\section{THE POSITIVE PREDICTIVE VALUE OF LYME ELISA FOR THE DIAGNOSIS OF LYME DISEASE IN CHILDREN}

\author{
Susan C. Lipsett, MD, * Nira R. Pollock, MD, PhD, $†$ \\ John A. Branda, MD, $\neq$ Caroline D. Gordon, BS, * \\ Catherine R. Gordon, BA, * Paul M. Lantos, MD, $\$$ \\ and Lise E. Nigrovic, $M D, M P H^{*}$
}

\begin{abstract}
By using a Lyme enzyme-linked immunosorbent assay (ELISA), we demonstrated that high ELISA index values are strongly predictive of Lyme disease. In children with clinical presentations consistent with Lyme disease, ELISA index values $\geq 3.0$ had a positive predictive value of 99.4\% (95\% confidence interval: $98.1-99.8 \%$ ) for Lyme disease, making a supplemental Western immunoblot potentially unnecessary.
\end{abstract}

Key Words: Lyme disease, Lyme ELISA, predictive value, pediatrics

Accepted for publication May 23, 2015.

From the *Division of Emergency Medicine, Boston Children's Hospital, Boston, Massachusetts; †Infectious Diseases Diagnostic Laboratory, Boston Children's Hospital and Division of Infectious Diseases, Beth Israel Deaconess Medical Center, Boston, Massachusetts; \$Department of Pathology, Massachusetts General Hospital, Boston, Massachusetts; and Divisions of $\S$ Pediatric Infectious Diseases and $\uparrow$ General Internal Medicine, Duke University School of Medicine, Durham, North Carolina.

This study was supported by a Boston Children's Hospital Pilot Research Grant (L.E.N.) and a National Center for Advancing Translational Sciences (NCATS) of the National Institutes of Health (NIH) KL2 TR001115 (P.M.L.). Branda reports receiving investigator initiated research grants from Immunetics, Inc., bioMérieux SA, Alere, Inc., and DiaSorin, Inc. and he was also a paid consultant to AdvanDx, Inc. All the other authors have no conflicts of interest to disclose.

Address for correspondence: Lise E. Nigrovic, MD, MPH, Division of Emergency Medicine, Boston Children's Hospital, 300 Longwood Avenue, Boston, MA 02115. E-mail: lise.nigrovic@childrens.harvard.edu.

Copyright (C) 2015 Wolters Kluwer Health, Inc. All rights reserved.

DOI: 10.1097/INF.0000000000000858

urrent serologic testing for Lyme disease follows a 2-tiered algorithm, an enzyme-linked immunosorbent assay (ELISA) followed by supplemental Western immunoblots if the first-tier ELISA is equivocal or positive. ${ }^{1}$ We investigated whether a quantitative Lyme ELISA results by itself could inform clinical decision making, without the need to wait for additional immunoblot results that may take several days. To this end, we performed a crosssectional study of children who had a Lyme ELISA performed by a single commercial laboratory over a 7-year period and investigated the predictive ability of Lyme ELISA index values across a range of cutpoints.

\section{MATERIALS AND METHODS}

\section{Study Design and Setting}

We performed a retrospective cross-sectional study at a single pediatric institution in a Lyme disease endemic area. We included all Lyme ELISA tests performed between January 1, 2007, and June 30, 2014. The institutional review board approved the study protocol with waiver of informed consent.

\section{Case Identification}

We identified all Lyme ELISA tests performed through an electronic query of the institutional data warehouse. We included all ELISA test results obtained from children younger than 21 years during the study period. Also, we included multiple test results obtained from the same patient. We selected Lyme ELISA tests with an index value $\geq 1.0$ (the cutpoint for an equivocal result according to the manufacturer's guidelines) and excluded all positive Lyme ELISAs without both immunoglobulin $\mathrm{G}(\operatorname{IgG})$ and immunoglobulin $\mathrm{M}$ (IgM) Western immunoblots performed from the same serum sample.

\section{Data Collection}

For all included Lyme ELISA tests, we abstracted the following patient-related data from the medical record onto a structured data form: demographics (age and gender), clinical symptoms, erythema migrans (EM) rash diagnosed by the treating clinician, ELISA index value and immunoblot results. All serologic Lyme disease tests obtained from the study institution were performed at a single commercial reference laboratory (ARUP National Laboratories, Salt Lake, UT, personal communication). This laboratory used the same Lyme ELISA and Lyme Western blot kits (MarDx, Trinity Biotech, Tray, Ireland) throughout the study period. As recommended by the manufacturer, the laboratory converted Lyme ELISA optical density values to index values by dividing the optical density value by a standardized factor. All specimens with Lyme ELISA index values $\geq 1.00+<1.20$ (equivocal) and $\geq 1.20$ (positive) were reflexively evaluated using both $\operatorname{IgG}$ and IgM Western immunoblots. The reference laboratory scored the index values that were evaluated using immunoblots based on the Centers for Disease Control and Prevention criteria. ${ }^{1}$

\section{Outcome Measure}

Our primary outcome was a case of confirmed Lyme disease defined as follows: either a physician-diagnosed EM rash or a positive 2-tiered Lyme serologic test (using criteria specified by the testing laboratory) in a patient with a clinical syndrome compatible with Lyme disease. A positive 2-tiered Lyme serology was defined as a Lyme ELISA index $\geq 1.0$ with either a positive IgG or a positive IgM immunoblot. ${ }^{1}$ A negative 2-tiered serology was defined as a Lyme index $<1.0$ or a Lyme index $\geq 1.0$ with negative $\operatorname{IgG}$ and $\operatorname{IgM}$ immunoblots. We defined the following as a clinical syndrome consistent with Lyme disease: early (single EM), early disseminated (multiple EM, cranial neuritis, meningitis, carditis) or late disseminated (large joint monoarticular arthritis). ${ }^{2}$ Children with nonspecific clinical presentations (eg, fever or fatigue) were considered negative for Lyme disease, even with a positive 2-tiered serology.

\section{Statistical Analysis}

We present continuous variables as medians with interquartile range and categorical variables as proportions. We selected the optimum cutpoint for the Lyme ELISA index value to discriminate Lyme disease from other causes of the patient's clinical findings and then calculated the positive predictive value. All data were analyzed using SPSS version 21.0 (SPSS Inc., Chicago, IL).

\section{RESULTS}

Of the 7289 Lyme ELISA tests performed, 1226 (17\%) had an index value $\geq 1.0$. Of these, we included the 1215 tests that were performed using supplemental Western blots (99\% of those with a positive or equivocal Lyme ELISA). The median patient age was 10.5 years (interquartile range, $7.1-14.4$ years). Lyme disease tests were performed for 87 children (7\% of tests sent) with EM rash, $258(21 \%)$ with suspected early disseminated disease, 695 (58\%) with suspected late disease and 175 (14\%) with nonspecific signs and symptoms.

Six hundred and eighty Lyme ELISAs (9\% of the ELISAs performed) were obtained from children with Lyme disease. Of these, the diagnosis of Lyme disease was as follows: 32 with EM rash alone, 22 with EM rash and positive 2-tiered Lyme serology 
TABLE 1. Classification of Patients by Lyme ELISA Across a Range of Cutpoints

\begin{tabular}{cccc}
\hline \hline & & \multicolumn{2}{c}{ Not Lyme Disease } \\
\cline { 3 - 4 } $\begin{array}{c}\text { Lyme ELISA } \\
\text { Cutpoint }\end{array}$ & $\begin{array}{c}\text { Lyme } \\
\text { Disease }(\%)\end{array}$ & $\begin{array}{c}\text { Negative } \\
\text { Western } \\
\text { Blot }(\%)\end{array}$ & $\begin{array}{c}\text { Positive } \\
\text { Western } \\
\text { Blot* }(\%)\end{array}$ \\
\hline $1.00(\mathrm{n}=1215)$ & $680(56)$ & $499(41)$ & $36(3)$ \\
$1.21(\mathrm{n}=953)$ & $651(68)$ & $268(28)$ & $34(4)$ \\
$2.00(\mathrm{n}=635)$ & $600(94)$ & $27(5)$ & $8(1)$ \\
$3.00(\mathrm{n}=468)$ & $465(99)$ & $3(1)$ & $0(0)$ \\
$4.00(\mathrm{n}=220)$ & $220(100)$ & $0(0)$ & $0(0)$ \\
\hline
\end{tabular}

*With a clinical presentation not consistent with Lyme disease.

and 626 with positive 2-tiered serology. Of those with positive 2-tiered serology, $144(20 \%)$ were positive by IgM immunoblot only, 295 (40\%) were positive by IgG immunoblot only and 209 $(40 \%)$ were positive by IgM and IgG Western immunoblot.

Increasing the Lyme ELISA index cutpoint increased the positive predictive value of the ELISA for the diagnosis of Lyme disease (Table 1). A Lyme ELISA index value $\geq 3.0$ was strongly predictive of Lyme disease [465 of 468; positive predictive value of $99.4 \%$ (95\% confidence interval: $98.1-99.8 \%$ )]. Of the 465 with Lyme disease, $33(7 \%)$ had early disease, $77(17 \%)$ had early disseminated disease and $355(76 \%)$ had late disease. The 3 children with a Lyme ELISA index $\geq 3.0$ and negative Western blot had knee monoarthritis and were diagnosed with non-Lyme inflammatory arthritis.

\section{DISCUSSION}

We performed a large cross-sectional study of children from a Lyme endemic region for whom 2-tiered Lyme disease serologic testing was obtained. A higher Lyme ELISA index value was more strongly predictive of confirmed Lyme disease as conventionally defined. For children with a Lyme ELISA index value between 1.0 and 2.9 and a clinical presentation compatible with Lyme disease, supplemental Western immunoblot testing should still be performed. However, as nearly all children with compatible clinical syndromes and a Lyme ELISA index $\geq 3$. 0 had Lyme disease, clinicians may be able to make initial clinical management decisions for these children without supplemental immunoblot results.

Clinicians caring for children with possible Lyme disease often need to make initial management decisions before results from 2-tiered Lyme serologic testing are available. Clinical prediction rules can assist decision making by estimating the risk of Lyme disease in children with facial palsy, ${ }^{3}$ meningitis ${ }^{4-6}$ or arthritis. ${ }^{7}$ However, the clinical presentations of Lyme disease and its mimics can overlap considerably. A rapid and accurate first-tier diagnostic test would facilitate early initiation of appropriate therapy while limiting the unnecessary invasive procedures. Antimicrobial therapy active against Borrelia burgdorferi could be promptly initiated for children with confirmed Lyme disease. Perhaps more importantly, in the appropriate clinical scenario, unnecessary invasive procedures such as arthroscopic joint washout for treatment of possible septic arthritis or hospital admission and parenteral antibiotic therapy could potentially be avoided for children with rapidly confirmed Lyme disease. ${ }^{8}$

Two-tiered Lyme disease serologic testing combines a sensitive ELISA with a specific supplemental Western immunoblot. We have demonstrated that, in a Lyme disease endemic area, a very high Lyme ELISA index value could be used to direct clinical decision making without a confirmatory Western immunoblot. The Lyme ELISA kit used by our reference laboratory is a moderate complexity diagnostic test that requires approximately 1 hour to obtain results. Although our study institution uses a reference laboratory, many institutions perform a Lyme ELISA on-site allowing for more rapid access to results. An optimal Lyme disease testing strategy for endemic regions may include a real-time Lyme ELISA, followed by confirmatory Western immunoblots for low-to-moderately positive Lyme ELISA tests.

Our study had several important limitations. First, because only Lyme ELISA tests with an index value $\geq 1.0$ had confirmatory Western blots, we cannot calculate the sensitivity and specificity of the Lyme ELISA. Second, we studied a single Lyme ELISA assay with numeric results based on the optical density values, which may not have a linear relationship with $B$. burgdorferi antibody titer. Therefore, our findings may not hold true for other Lyme ELISA assays. ${ }^{9}$ Third, a positive IgM immunoblot may be falsely positive, particularly if the patient's symptoms have been present for more than 1 month. ${ }^{10}$ However, when we reclassified children with a positive IgM alone with more than 1 month of symptoms as not having Lyme disease, the Lyme ELISA positive predictive value dropped only slightly (data not shown). Fourth, we conducted our study in a Lyme disease endemic area. Children from a geographic area with a lower prevalence of Lyme disease or outside the typical season may require supplemental serologic testing even when the Lyme ELISA index value is significantly increased. ${ }^{11,12}$ Finally, we recognize that our findings in a pediatric population may not apply to adult patients.

The burden of Lyme disease continues to increase annually. On the basis of a 2008 survey of 7 large commercial laboratories, investigators estimated an annual incidence of Lyme disease in the hundreds of thousands each year, at a cost of 500 million U.S. dollars for diagnostic testing. ${ }^{13}$ Appropriate clinical utilization of 2-tiered Lyme serologic testing, including limiting unnecessary Western immunoblots for children from Lyme disease endemic areas, could limit unnecessary expenditures. Newer serologic assays (eg, VlsE or C6 peptide) seem to have higher sensitivity in early Lyme disease $^{14}$ but have not yet been evaluated in children. Future evidencebased guidelines will need to provide clinical guidance about the optimal utilization of available Lyme diagnostics.

\section{REFERENCES}

1. Recommendations for test performance and interpretation from the Second National Conference on Serologic Diagnosis of Lyme Disease. $M M W R$ Morb Mortal Wkly Rep. 1995;44:590-591.

2. Wormser GP, Dattwyler RJ, Shapiro ED, et al. The clinical assessment, treatment, and prevention of lyme disease, human granulocytic anaplasmosis, and babesiosis: clinical practice guidelines by the Infectious Diseases Society of America. Clin Infect Dis. 2006;43:1089-1134.

3. Nigrovic LE, Thompson AD, Fine AM, et al. Clinical predictors of Lyme disease among children with a peripheral facial palsy at an emergency department in a Lyme disease-endemic area. Pediatrics. 2008;122:e1080-e1085.

4. Avery RA, Frank G, Glutting JJ, et al. Prediction of Lyme meningitis in children from a Lyme disease-endemic region: a logistic-regression model using history, physical, and laboratory findings. Pediatrics. 2006;117:e1-e7.

5. Garro AC, Rutman M, Simonsen $\mathrm{K}$, et al. Prospective validation of a clinical prediction model for Lyme meningitis in children. Pediatrics. 2009;123:e829-e834.

6. Cohn KA, Thompson AD, Shah SS, et al. Validation of a clinical prediction rule to distinguish Lyme meningitis from aseptic meningitis. Pediatrics. 2012;129:e46-e53.

7. Deanehan JK, Kimia AA, Tan Tanny SP, et al. Distinguishing Lyme from septic knee monoarthritis in Lyme disease-endemic areas. Pediatrics. 2013;131:e695-e701.

8. Deanehan JK, Nigrovic PA, Milewski MD, et al. Synovial fluid findings in children with knee monoarthritis in lyme disease endemic areas. Pediatr Emerg Care. 2014;30:16-19.

9. Hansmann Y, Leyer C, Lefebvre N, et al. Feedback on difficulties raised by the interpretation of serological tests for the diagnosis of Lyme disease. Med Mal Infect. 2014;44:199-205. 
10. Seriburi V, Ndukwe N, Chang Z, et al. High frequency of false positive IgM immunoblots for Borrelia burgdorferi in clinical practice. Clin Microbiol Infect. 2012;18:1236-1240.

11. Lantos PM, Brinkerhoff RJ, Wormser GP, et al. Empiric antibiotic treatment of erythema migrans-like skin lesions as a function of geography: a clinical and cost effectiveness modeling study. Vector Borne Zoonotic Dis. 2013;13:877-883.

12. Lantos PM, Branda JA, Boggan J, et al. Poor positive predictivie value of Lyme disease serologic testing in an area of low disease incidence. Clin Infect Dis. [published online ahead of print July 20, 2015]. doi: 10.1093/cid/ civ584.

13. Hinckley AF, Connally NP, Meek JI, et al. Lyme disease testing by large commercial laboratories in the United States. Clin Infect Dis. 2014;59:676-681.

14. Branda JA, Linskey K, Kim YA, et al. Two-tiered antibody testing for Lyme disease with use of 2 enzyme immunoassays, a whole-cell sonicate enzyme immunoassay followed by a V1sE C6 peptide enzyme immunoassay. Clin Infect Dis. 2011;53:541-547.

\section{INVASIVE FUNGAL INFECTIONS IN PEDIATRIC ACUTE MYELOGENOUS LEUKEMIA}

\author{
Stéphane Ducassou, MD, * Delphine Rivaud, MD, * \\ Anne Auvrignon, $M D, \uparrow$ Cécile Vérité, $M D$, * \\ Yves Bertrand, $M D, P h D,+$ Virginie Gandemer, $M D, P h D, \S$ \\ and Guy Leverger $M D, P h D+$
}

\begin{abstract}
This study investigates invasive fungal infections (IFIs) in patients with acute myelogenous leukemia who were included in the Enfant Leucemie Aigue 02 protocol between 2005 and 2011. Among 387 patients, 15 had aspergillosis, 9 had candidiasis, and 2 had mucormycosis. The most frequent localization of IFIs was in the lungs. It was found that after a median of 34 months, 2 deaths were attributable to IFI without any difference in survival between the groups with and without IFI.
\end{abstract}

Key Words: fungal infection, acute leukemia, childhood, neutropenia

Accepted for publication May 23, 2015.

From the *Unité d'Oncologie Hématologie Pédiatrique, Hôpital des Enfants, Groupe Hospitalier, Pellegrin, CHU de Bordeaux, Bordeaux Cedex, France; †Service d'Onco-Hématologie Pédiatrique, Hôpital Armand-Trousseau, Paris Cedex, France; IInstitut d'Hématologie Oncologie Pédiatrique, CHU Lyon, Lyon Cedex, France; and §Unité d'Hémato-Oncologie Pédiatrique, CHU Hôpital Sud, Rennes Cedex, France.

The authors have no funding or conflicts of interest to disclose.

Address for correspondence: Stéphane Ducassou, MD, Unité d'Oncologie Hématologie Pédiatrique, Hôpital des Enfants, Groupe Hospitalier Pellegrin, CHU de Bordeaux, 33076 Bordeaux Cedex, France. E-mail: stephane.ducassou@chu-bordeaux.fr.

Copyright (C) 2015 Wolters Kluwer Health, Inc. All rights reserved.

DOI: $10.1097 /$ INF.0000000000000875

nvasive fungal infections (IFIs) affect the prognosis of pediatric patients with oncological and hematological malignancies. Many children recover nowadays from hematological malignancies because of increased treatment intensity, but the number of infections, especially fungal complications, has increased. ${ }^{1}$ Various risk factors for IFIs have been identified. ${ }^{1}$ Among the hematological malignancies, acute myelogenous leukemia (AML) is a subgroup at risk. $^{2}$ Despite the advent of new antifungal agents and the development of new diagnostic methods, mortality due to fungal infections remains high, ranging from $12 \%$ to $18 \%{ }^{1,2}$

The Fourth European Conference on Infections in Leukemia recommendations provide guidelines for the management of patients in a manner that is homogeneous and evidence based. ${ }^{3}$ Using data from a national multicenter retrospective study, we report the occurrence of IFIs in a cohort of pediatric AML. This article reports the characteristics (epidemiology, risk factors, clinical presentation, diagnosis, therapeutic management and evolution) of IFIs in children treated for AML since the advent of the new antifungal agents. We also conducted a comparative study with the rest of the cohort who did not develop IFIs.

\section{PATIENTS AND METHODS}

This retrospective multicenter study included patients less than 18 years of age who were treated for AML in the ELAM 02 protocol between March 16, 2005 and March 7, 2011 and who developed IFIs according to the criteria of the EORTC/MSG group (European Organization for Research and Treatment of Cancer/Invasive Fungal Infections Cooperative Group and the National Institute of Allergy and Infectious Diseases Mycoses Study Group), ${ }^{4}$ which contain 3 categories: proven, probable and possible.

The treatment of AML comprised an induction course and 3 consolidation courses for nontransplanted patients taking cytarabine + mitoxantrone, high-dose cytarabine + amasacrine, cytarabine + VP16 + daunorubicine and high-dose cytarabine + asparaginase, respectively. After these 4 courses, there is a randomization for the addition or not of maintenance therapy with Interleukin 2 for patients in complete remission. For induction, it is recommended to isolate the patient in a sterile room for a median duration of 35 42 days, which corresponds to the duration of aplasia. In case of refractory disease at day 21 , the isolation can be extended till the beginning of the first consolidation course, whatever may be the neutrophil count of the patient.

Demographic, clinical, biological, radiological and therapeutic data were collected prospectively in the protocol database and retrospectively from patient records. Patients with digestive colonization, isolated cutaneous lesions and posttransplant IFI were excluded. Statistical analysis of baseline characteristics was performed with SAS software V9.2 (SAS Institute Inc., Cary, NC) using a $\chi^{2}$ test or Fisher exact test for categorical variables and a nonparametric Wilcoxon test for quantitative variables. Survival analysis was performed with the R package version 2.7.2. Survival curves were obtained with the Kaplan-Meier method. Survival rate between 12 and 24 months and survival comparisons were calculated with the log-rank test.

\section{RESULTS}

Of 387 patients enrolled between 2005 and 2011, there were 26 cases of IFI, that is, an incidence of $6.7 \%$. The median age at diagnosis of AML was 12 years ( 5 months to 17 years). The sex ratio (male/female) was 1.36 . The mean duration of neutropenia (absolute neutrophil count between $0.5 \times 10^{9}$ and $1 \times 10^{9}$ per liter) preceding IFI was 17 days (7-44) and mean cumulative neutropenia was 23.5 days (7-97). Only 3 patients were nonneutropenic at the time of diagnosis of IFI, including 2 with hepatosplenic candidiasis. All patients received a wide-spectrum antibiotherapy for a median of 25.5 days (8-61.6) before the diagnosis of IFI.

Comparison of populations with or without IFI did not reveal any specific risk factor (Tables 1 and 2). Although the mean age of the group with IFI was higher, there was no significant difference in age between the groups with and without IFI. On the other hand, there were more cases of undocumented fever at diagnosis in the group without IFI $(P=0.05)$. There were 15 cases of aspergillosis (only 3 species were identified with 2 Aspergillus fumigatus and 1 Aspergillus niger), 9 cases of candidiasis and 2 cases of mucormycosis, divided into proven (12), probable (6) and possible (8), which were diagnosed mainly during the intensive phase (16 during induction and 5 during the third consolidation phase). All patients were febrile at diagnosis of IFI, 8 had cough, 7 had chest pain, and 6 had dyspnea. An isolated or associated pulmonary localization was the most frequent presentation (20 of 26). 